\title{
Multi Electrical Machine Pre-Design tool with error handling and machine specific advanced graphical design aid features based on Modelica
}

\author{
Tomasz D. Michaski ${ }^{1} \quad$ Antoni Garcia Espinosa ${ }^{2} \quad$ Jordi-Roger Riba Ruiz $^{3} \quad$ Luís Romeral Martínez $^{4}$ \\ ${ }^{2,3}$ Departament of Electrical Engineering, Universitat Politècnica de Catalunya, Spain, \\ garciaedee.upc.edu, riba@ee.upc.edu \\ ${ }^{1,4}$ Departament of Electronic Engineering, Universitat Politècnica de Catalunya, Spain, \\ tomasz.michalski@mcia.upc.edu, luis.romeral@mcia.upc.edu
}

\begin{abstract}
This paper presents a design tool for Induction Machines, Permanent Magnet Synchronous Machines, Externally Excited Synchronous Machines and Switched Reluctance Machines. This software, based on Modelica language, is able to provide full dimensioning (cross and axial section measures) and operation characteristics according to mechanical and electrical requirements set as inputs. The tool is able to perform error handling, which informs a designer about unfeasible designs and gives clues about the possible errors. Both aspects of the tool GUI and scripts provide help files and code explanation in order to re-use the tool and improve library's functionalities.
\end{abstract}

Keywords: Modelica, design tools, electrical machines, SMPMSM, IPM, Synchronous Machine, SRM, Efficiency Map.

\section{Introduction}

Electrical motors use is spreading in new fields and they are replacing other actuators because of their performance, power, torque density and reliability. However, in order to integrate these new components into a system engineering design process, the models of electrical machines must be pre-evaluated and designed for specific uses.

In the past years several phenomes have changed the panorama regarding electrical machines typology. Regarding motors using magnets: appearance and proliferation of Surface Mounted Permanent Magnet Synchronous Motors, internal Permanent Magnet Motor Family, emerge and intensive development due to magnet's price raises (substitution of rare earth magnets by ferrite magnets) and increased power density requirements (keeping rare earth magnets in geometrical configurations meant to achieve high airgap flux). Regarding externally excited Synchronous Machines [1]-[2], its ability to work in four quadrants and to work under very high temperatures (where magnet motors get demagnetized) made them resurge in micro generation, both in land and aviation fields. These new appearances did not pull back Induction
Machines because of, as in Switched Reluctance Motors, its low construction and operation costs are still an advantage [3].

The electrical machine design process has also changed due to the reduction of FEA costs and high demand of motors for specific purposes. Both phenomena made it possible to start developing specifically optimized motors out of catalogue increasing the added value of small companies.

Not only design process has changed, but its implementation and tests before construction. The introduction of systems engineering design in Product Lifecycle Management requires parametrized models of all its components. Modelica not only fits in that spot but also allows multi-parametric, multi-physics implementation of such models for both: steady state and transient simulations [4].

The work presented in this paper is the first Modelica implementation of pre-design tool able to provide the data required to create such models in order to integrate the specific machine size and properties into system models and is intended to work under Clean Sky [5] European Initiative therefore the library along with help files, and manuals are free to use. This novel tool in the Modelica society also leaves a possibility to be used with other already developed open and commercial libraries i.e. outputs of predesign sizing algorithms can be passed as inputs to electrical machines advanced and complex models such as the Actuation 2015 project [6].

In the cases where, in a system model, an electrical machine is required, the user is force to select from pre-designed models (with restricted electromechanical sizes) or manually calculate the machine parameters for the purpose he is modelling for. With this new tool, added to the Modelica chain, and given designer's specifications, insert the specific machine he needs to work with.

\section{Basic Pre-design tool}

\subsection{Scope}

Basic pre-design tool is conceived as a series of Modelica functions meant to return physical, 
geometrical, electrical and magnetic properties as a starting point of an electrical machine's design. These scripts can be run from within Modelica or by a third party GUI able to call Modelica scripting. The program is able to pre-design Induction Motors (IM), Surface Mounted Permanent Magnet Synchronous Motors (SMPMSM), Internal Permanent Magnet Synchronous Motors (IPM) in their Spoke (embedded and nonembedded magnets), V-Shape and Planar configurations. It also performs pre-sizing for Switched Reluctance Motors (SRM) and externally exited Synchronous Machines (Syn). Each machine runs its own pre-sizing algorithm even though some of the subfunctions are shared across machines like winding factor calculation or number of gauged turns.

Since SMPMSM and IM machines have been deeply analyzed, this work is more focused in the IPM family, SRM and Synchronous machines.

For IPM family the program returns all cross section and axial section dimensions for stator, rotor, magnets shaft and hub if required. Following outputs are provided: moments of inertia of stator, rotor and total, mass of stator, rotor, total mass including housing as well as masses separated by materials like magnet mass, magnetic steel mass and copper. Subsequent are inductances and phase resistance based on desired working temperature. The tool also includes saturation factor of airgap flux, Back-EMF, number of turns per phase and final obtained power. With this data an efficiency map including losses is also calculated and performed.

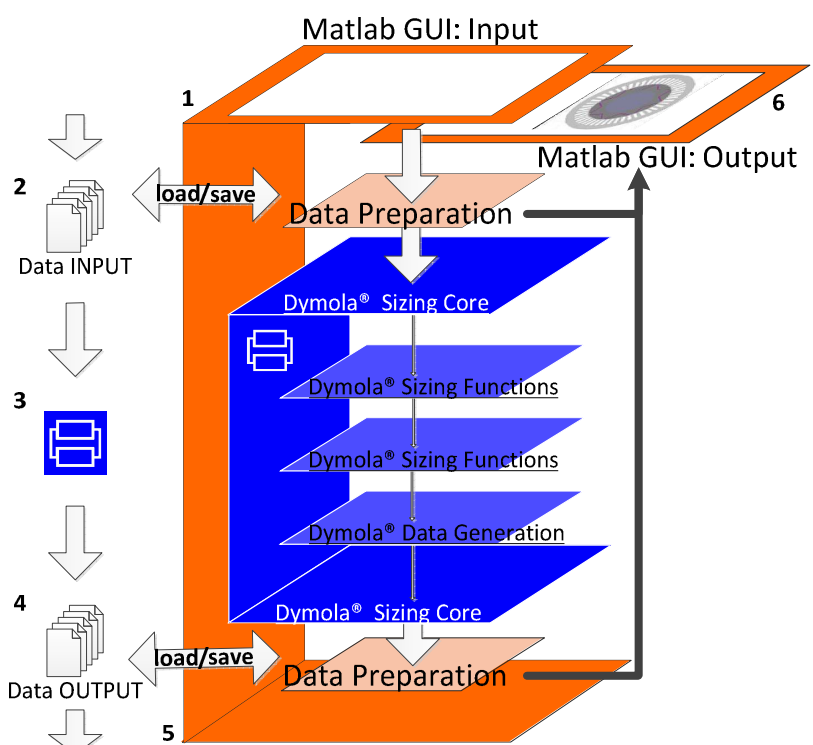

Figure 1. Layer structure of the GUI and Modelica presizing core code.

\subsection{Internal Operation and Process}

The sets of inputs are large and vary from one machine to another. Among them there are first tier and second tier inputs. First tier are basic indispensable values for pre-sizing, and second tier those pre-sizing tool can automatically set if left empty or its influence is minor. For the first tier inputs power, efficiency, current or voltage are some of the electrical parameters required. Airgap flux or magnetic steel or magnet properties are among the magnetic parameters. Part of the second tier inputs would be stator shoe tooth separation or shaft diameter external or extra length for housing. Because of the amount of inputs and its diversity an external input GUI was developed.

As Figure 1 shows, this GUI is a cover over Modelica functions. It allows opening and saving machines and has its own help documentation. When the inputs are set, the GUI prepares the data and sends it to Modelica by means of a callback of the sizing scripts which starts performing the sizing.

In this stage the several Modelica algorithms perform pre-sizing. When finished data is structured and send back to the GUI. In this stage is when motor can be saved with both input and output data. Also in this stage is when output data can be analyzed with the provided output sub tools.

\subsection{Modelica Sizing Core}

Modelica sizing core consists of five principal algorithms which perform sizing code for each machine: IM, PMSM, IPM, SRM and SYN. As shown in Figure 3, all of them utilize other common functions: winding (returns the winding factor of the fundamental frequency), CmecCALC (interpolates mechanical constant), htrmodif (recalculates slot height based on trapezoidal slot to one based on squared slot), hexapack (obtains a number of conductors per area following a hexagonal pattern), quadrapack (obtains a number of conductors per area following a square pattern), MassandMOI (calculates mass and moments of inertia), Resistance (returns resistance per phase), MassandMOISYN (calculates mass and moments of inertia of Synchronous machine), round_Nph (rounds number of turns per phase), cartdistance (returns distance between two points in Cartesian coordinates), Rotation (performs basic algebraic rotation in Cartesian coordinates), Reflection (performs basic algebraic reflection through axis situated at the angle position), feasibleregion (determines if angle is feasible for SRM motor design), Torquevs Speed (returns torque vs speed vs efficiency map as a matrix), Inductances (calculates d-q inductances), Rotorshape (generates a valid rotor geometry for IPM machine), MAGNETdb (returns properties of selected permanent magnet) and ellipse (finds ellipse from five points and returns its center).

Every main sizing function writes data in special format using DataFiles, writemATRIX and Utilities.Streams.print functions. After that 
machine structure is read by the external graphic user interface.

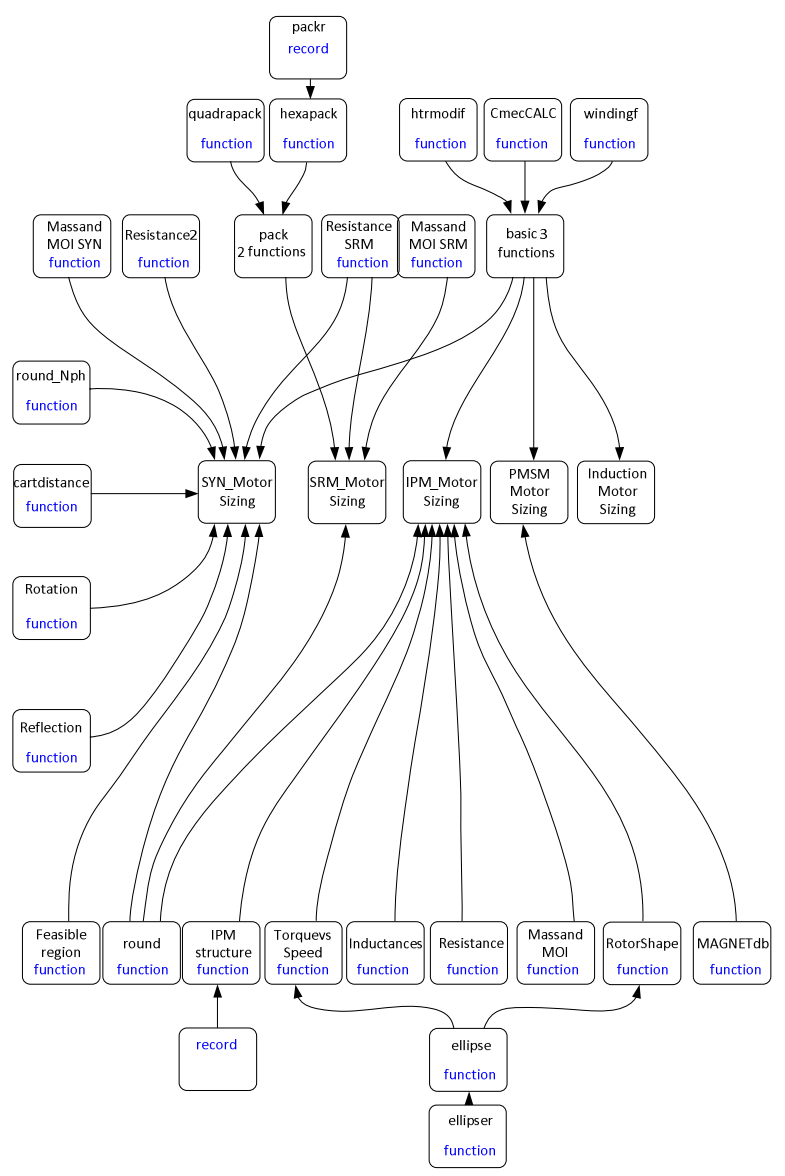

Figure 3. Modelica sizing relation tree of functions.

\subsection{MATLAB GUI}

Graphic User Interface was programmed in MATLAB Guide tool. This was done because it offers a good trade between complex plot representations and an appeal interface without the use of complex or third party APIs. All of the results from Dymola pre-sizing are decoded and interpreted on this stage.

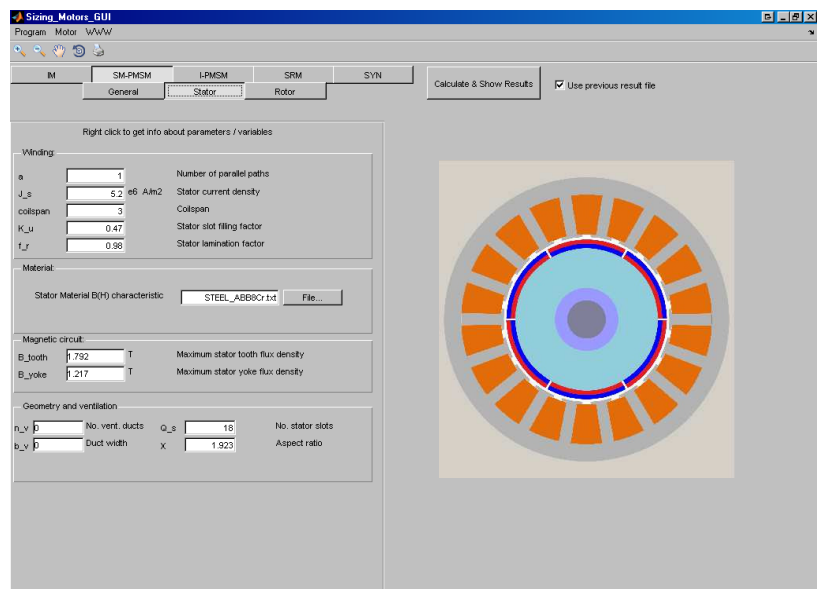

Figure 4. Pre-sizing GUI for SMPMSM inputs. Each variable contains a brief description when prompted.

The Graphical User Interface software is presented in. Figure 2 and Figure 4. This was done because currently Dymola serves as a simulation environment and there are no tools publically available for generation of the graphical user interface [7]. However user is expected to performs various pre-design runs, inspect and print results if necessary.

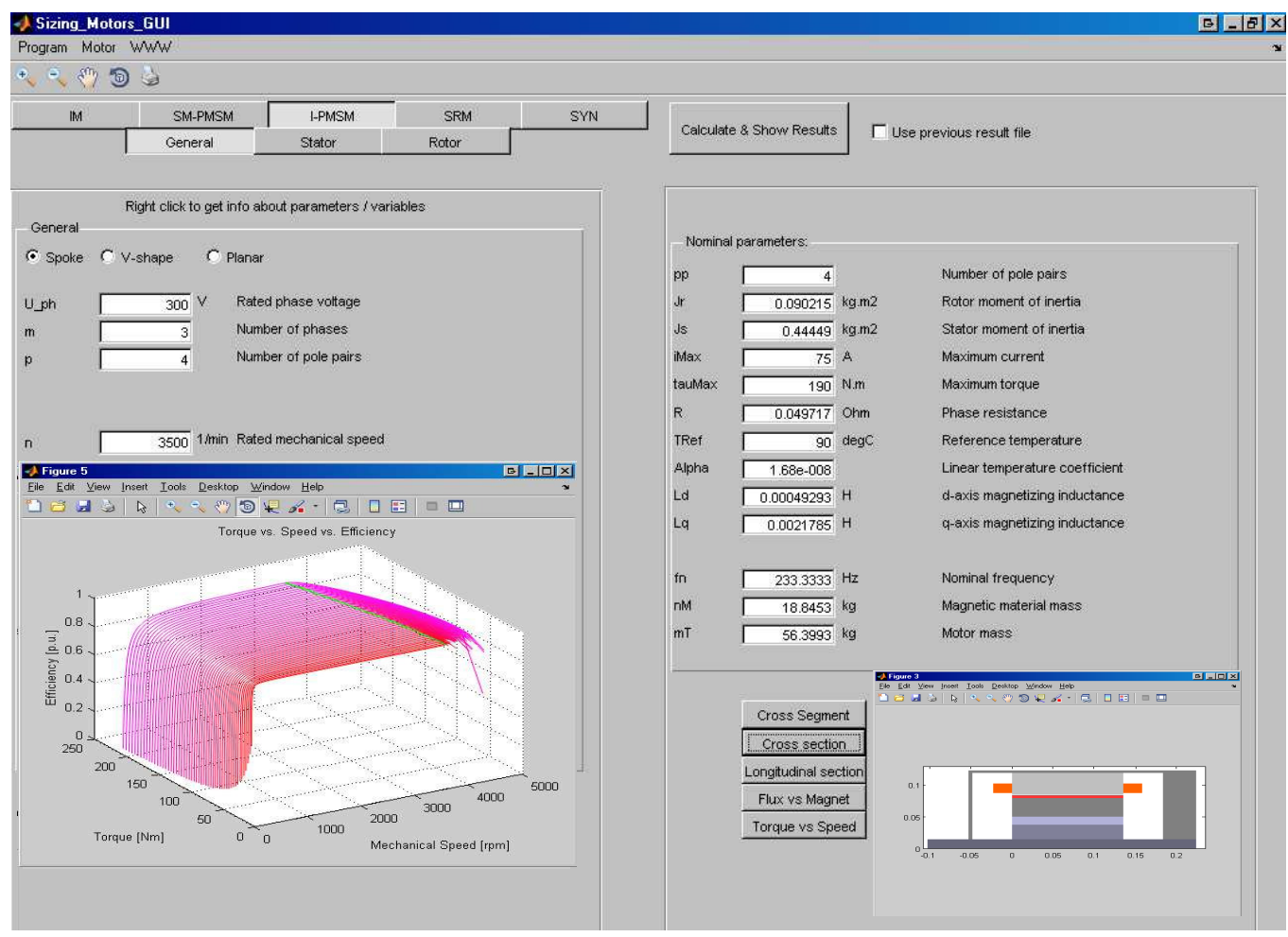

Figure 2. Example of Input/Output GUI with some windowed sub tools for the IPM machine. 


\section{Example of Use}

V-shape Internal Permanent Magnet will be used in the example to show most functionalities of the tool including the graphic error handling, console messages and height vs. PM. Figure 5 shows Modelica's sizing core for this kind of machines.

When inputs are set and sent to sizing core several data is calculated before the actual sizing procedure starts, for example, if not set, shaft and hub diameters. Pole shoe height is determined depending on desired power.

And finally total length and polar pitches. Then stator sizing starts taking into account flux saturation by means of a flux form factor coefficient corrector. Desired fluxes in airgap, tooth and yoke and winding configuration will determine the final size, number of conductors and saturation.

Then rotor calculation starts. It is worth noting that IPM rotor can be very complex and incur in several parts collisions, this it is explained in more detail in section 3.2. That is why rotor is sized by two main loops, one for the geometrical and the other for the magnetic considerations. If rotor is physically impossible program aborts, if not, then geometry of magnet, its position and reluctance paths are send to the magnetic circuit solving algorithm that will determine if the magnet quantity is enough to sustain the airgap flux, if not, magnet height is increased and rotor data is send back to geometry validation step.

\subsection{First input set}

The desired phase voltage, phase current, power, rated torque, rated speed, power factor and efficiency (which later on are corrected). Number of phases, number of poles, form factor as well as winding properties are the main parameters for design process. During this initial round it is recommended to use default values for flux densities. Desired Airgap, Stator Tooth and Yoke Flux Densities are required, and, as second tier, geometric properties like shaft diameter and length, slot opening, or filling and stack factors. Although these parameters can have a great influence in machine's design, they have to adapt to electrical and mechanical requirements not the other way around.

Further iterations can be used to refine input parameters if output geometry or features doesn't look reasonable, for example big yokes or too narrow tooth tip. Then the script is launched and performs sizing. Usually a set of inputs leads to impossible geometry or magnetic circuit, which is why an error handling system was developed.

\subsection{Error Handling: Console and Graphics}

Internal PMSM incorporates very complex rotor geometry because of the number of independent elements that conforms it: rotor shaft, rotor hub, air barriers, wedge dimensioning and magnets size and positioning [8]. Figure 6 shows geometry used to obtain geometry.

It is not suitable for an ease of use of pre-design to ask for more than twenty parameters associated only to those elements. The basic pre-design tool is able to position the magnet with four parameters, cover factor, desired wedge, top air barrier length and V-shape angle. Summed to the diameters automatically calculated magnets are positioned and all associated elements obtained (Figure 6): reluctance path lengths and widths, centers of gravity and areas. This is thanks to a parametrized model of its geometry.

Outfits and collisions are detected by rules of relative position of the aforesaid parametrization by means of

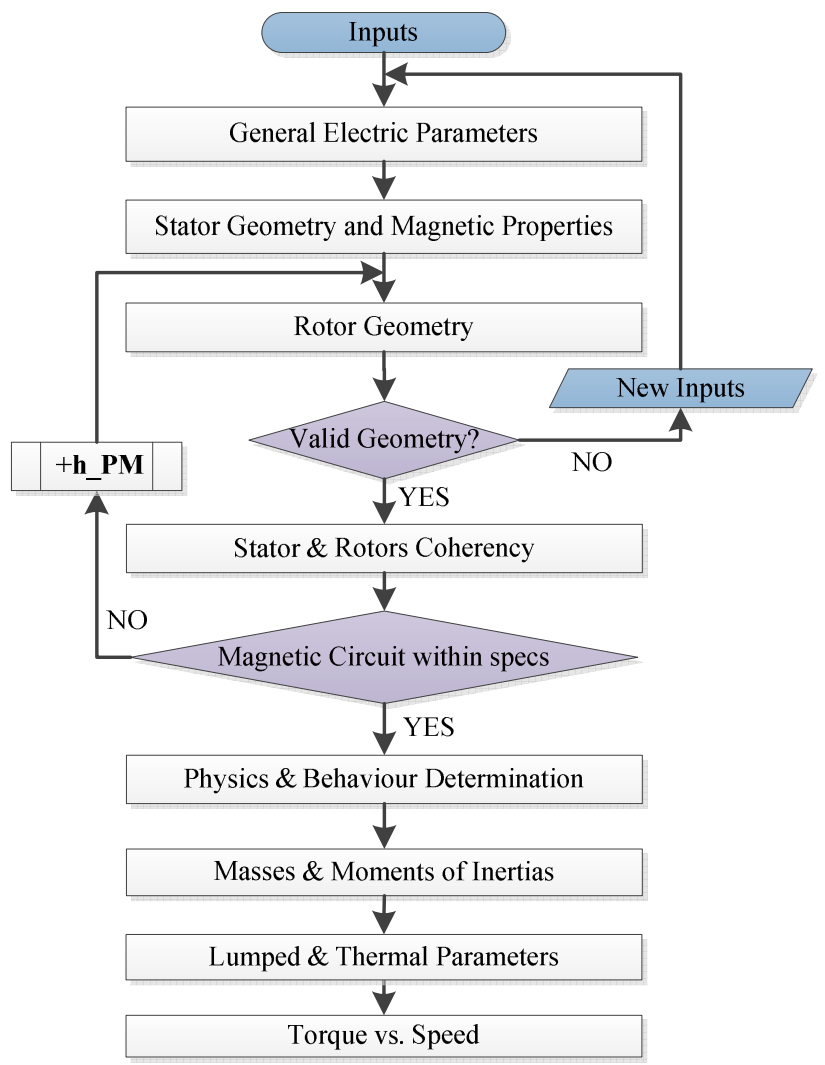

Figure 5. Sizing-core flow chart for IPM machines.

points. For example, alpha_v cannot be smaller than half pole pitch, which is the same to say that Point5 would be further than machine center.

Another typical example is when $\mathrm{V}$-shape angle is to narrow, in that cases distance of Point4 is inferior than shaft radius which leads to a collision. The program is able to detect these collisions and report them in both graphical representation and console. Console messages appear at the very same moment that the problem is generated, for graphical representation, Modelica has to return the control to the GUI which will call the cross section function graphical representation. 


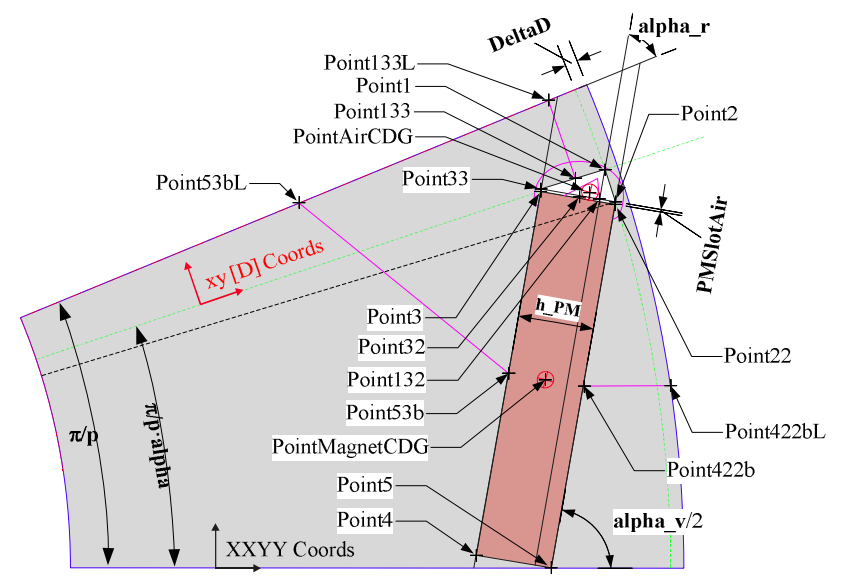

Figure 6. Semi pole graphical representation of the geometrical assistant points used to fit rectangular magnets inside rotor. This is only valid for $\mathrm{V}$-shape with only one air barrier in top.

An example of the console messages are as follows:

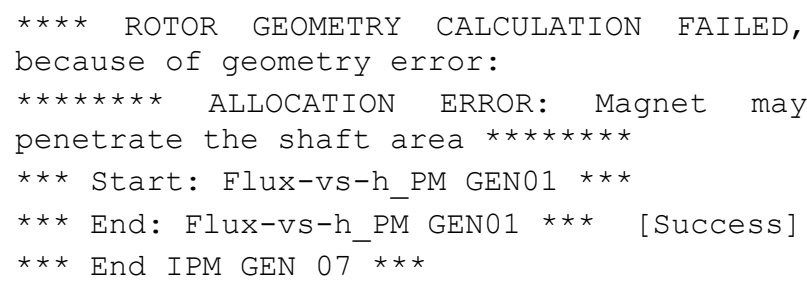

Each geometry message errors return, when possible, the nature of the sizing error, as well, each function returns and START-END messages.

And the graphical representation is as shown in Figure 7. The plot is not rasterized; zooming to any area will not carry a loss of detail. Collision information is saved in motor structure allowing other third party applications to perform its own interpretation of collisions.

\subsection{Final Iteration and results comparison}

Problem with shaft diameter can be changed by two means, one increasing V-shape angle and the other reducing shaft diameter. The second solution becomes a better approach when it comes to reduce the impact in machine properties. Once enough space for magnets is given the final motor is generated.

By properly setting inputs it is possible to achieve close to FEA 2D results, this is shown in Table 1.

It is not the aim of the tool, and it cannot, to substitute FEA in design process. This level of basic pre-design does not allow taking into account complex reluctance network effects and saturation effects, which are directly related to $\mathrm{d}-\mathrm{q}$ inductance values. However, being able to achieve these results it is especially useful when designing new machines between two well-known sizes or rated powers, or during optimization processes.

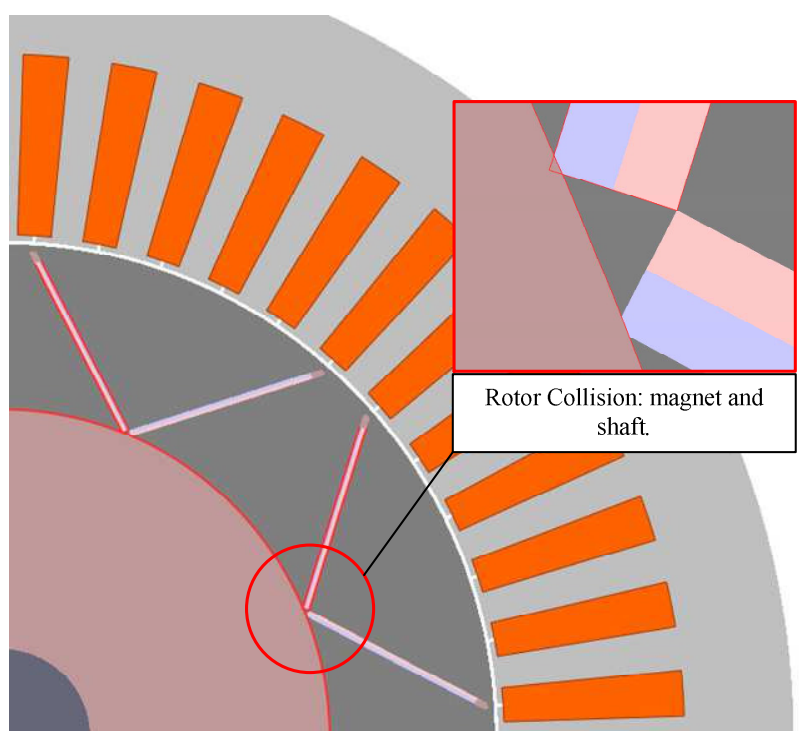

Figure 7. Graphical output error handling, remarks in red contours and dim colors of parts colliding. Zoom can be performed in figures to see the detailed problem.

Table 1. Comparison between FEA and pre-design too.

\begin{tabular}{|l|l|l|l|}
\hline FEM/Real & Parameter & $\begin{array}{l}\text { Pre-design } \\
\text { Calculated }\end{array}$ & $\begin{array}{l}\text { Relative } \\
\text { Error }\end{array}$ \\
\hline 97,78 & Total Mass [Kg] & 100,35 & $2,6 \%$ \\
\hline 0,08 & $\begin{array}{l}\text { Rotor Moment of } \\
\left.\text { Inertia [Kg· }{ }^{2}\right]\end{array}$ & 0,0832 & $2,7 \%$ \\
\hline 1,51 & $\begin{array}{l}\text { Stator Moment of } \\
\left.\text { Inertia [Kg } \cdot \mathrm{m}^{2}\right]\end{array}$ & 1,504 & $0,2 \%$ \\
\hline 0,088 & $\begin{array}{l}\text { Phase Resistance } \\
{[\Omega]}\end{array}$ & 0,0943 & $6,8 \%$ \\
\hline 0,0021 & $\begin{array}{l}\text { Direct Axis } \\
\text { Inductance [H] }\end{array}$ & 0,0026 & $25,4 \%$ \\
\hline 0,0091 & $\begin{array}{l}\text { Quadrature Axis } \\
\text { Inductance [H] }\end{array}$ & 0,01183 & $29,4 \%$ \\
\hline 0,0048 & $\begin{array}{l}\text { Final Flux in } \\
\text { Airgap [Wb] }\end{array}$ & 0,0044 & $8,0 \%$ \\
\hline 161,00 & $\begin{array}{l}\text { Back Electro } \\
\text { Motive Force [V] }\end{array}$ & 153,73 & $4,5 \%$ \\
\hline 48915,00 & $\begin{array}{l}\text { Rated } \\
\text { Mechanical } \\
\text { Power [W] }\end{array}$ & 48939,56 & $0,1 \%$ \\
\hline 282,00 & $\begin{array}{l}\text { D outer stator } \\
{[\text { mm] }}\end{array}$ & 292,59 & $3,8 \%$ \\
\hline 184,00 & $\begin{array}{l}\text { D outer rotor } \\
{[\text { mm] }}\end{array}$ & 186,52 & $1,4 \%$ \\
\hline 75,00 & Length [mm] & 75,95 & $1,3 \%$ \\
\hline 30,00 & Slot Height [mm] & 30,72 & $2,4 \%$ \\
\hline 6,50 & $\begin{array}{l}\text { Tooth Width } \\
{[\text { mm] }}\end{array}$ & 6,05 & $7,0 \%$ \\
\hline 18,40 & Yoke Height & 21,72 & $18,0 \%$ \\
\hline
\end{tabular}




\begin{tabular}{|l|ll|l|l|}
\hline & {$[\mathrm{mm}]$} & & \\
\hline 27,00 & $\begin{array}{l}\text { Magnet } \\
{[\mathrm{mm}]}\end{array}$ & Length & 27,69 & $2,5 \%$ \\
\hline 5,00 & $\begin{array}{l}\text { Magnet } \\
{[\mathrm{mm}]}\end{array}$ & Height & 5,15 & $3,0 \%$ \\
\hline
\end{tabular}

\subsection{Output sub-tools of the GUI}

Raw data of the pre-design requires interpretation especially when the volume of information is high. In order to present pre-sizing outputs in an easy going way several tools were developed. Some are specific for each machine and some are shared across all packages.

All outputs depart from the output section of the GUI where basic sizing information is presented. An example of the output GUI is shown in Figure 8. Each machine has its own outputs sets, interface and tools.

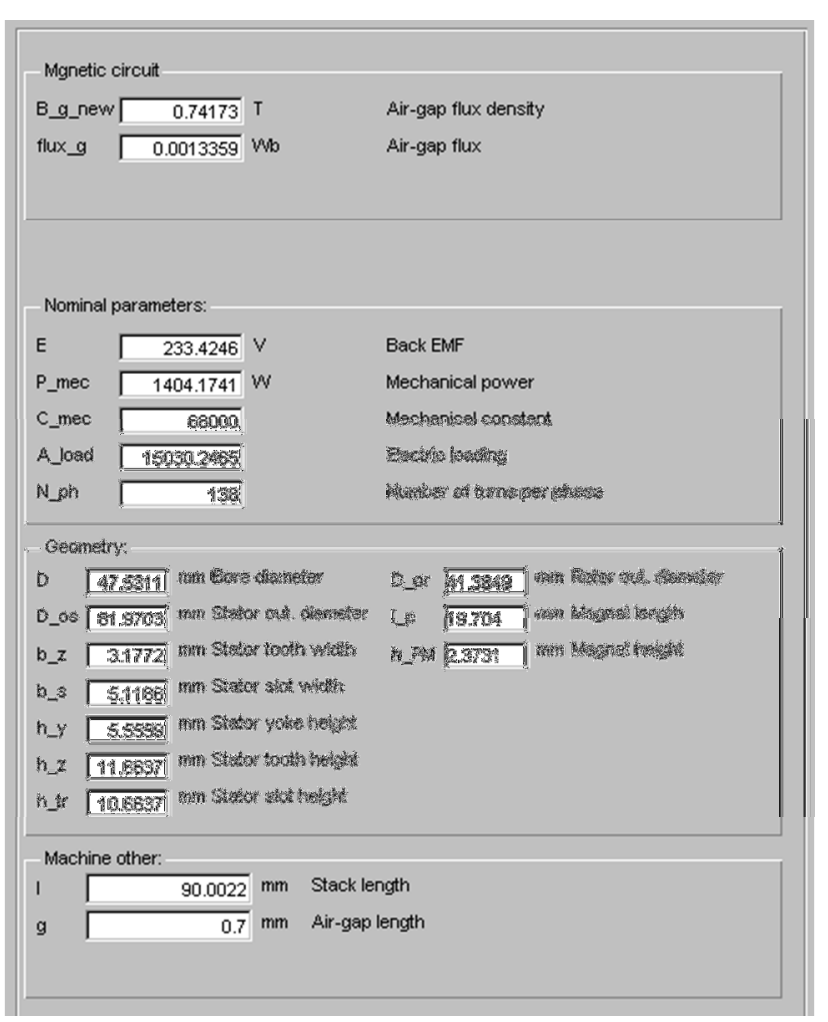

Figure 8. Screenshot of SM-PMSM pre-design outputs.

The most reliable and informative way to read dimensions relationship is to generate blueprints, but given dimension's magnitude are already computed, the use of scaled colored representations is more valuable. It is able to return fast qualitative information as well as raw dimensions. That is why scaled cross and axial sections of the machine were implemented in the output stage. Figure 9 contains two examples: for Synchronous Machine and Switched Reluctance Machine. This allows a fast eye check of dimensions and proportions giving almost instantly clues about what can fail in a design. If the design is considered good the designer can directly import output values to his design table.

For SRM specific case a feasible region chart was elaborated [9]. An example of it can be seen in Figure 10. This chart locates the motor in a five conditions diagram. Three are related to the good behavior of the motor, and two to physical limitations given the desired number of poles for stator and rotor.
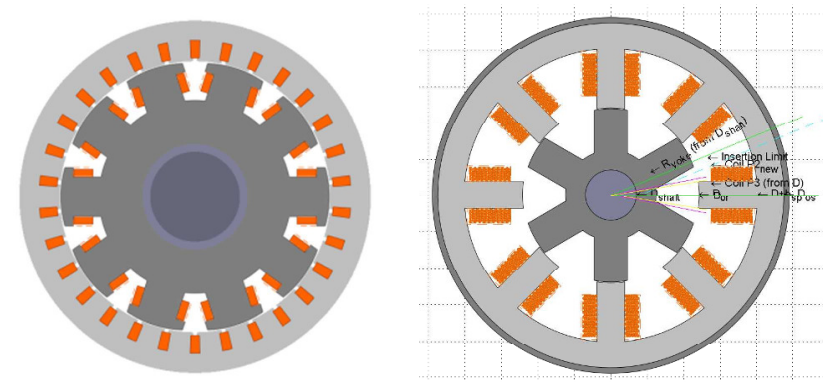

Figure 9. Example of two cross sections of different machines with different graphical detail set. Left, Salient Pole Synchronous Machine. Right, Switched Reluctance Motor with dimensional aids.

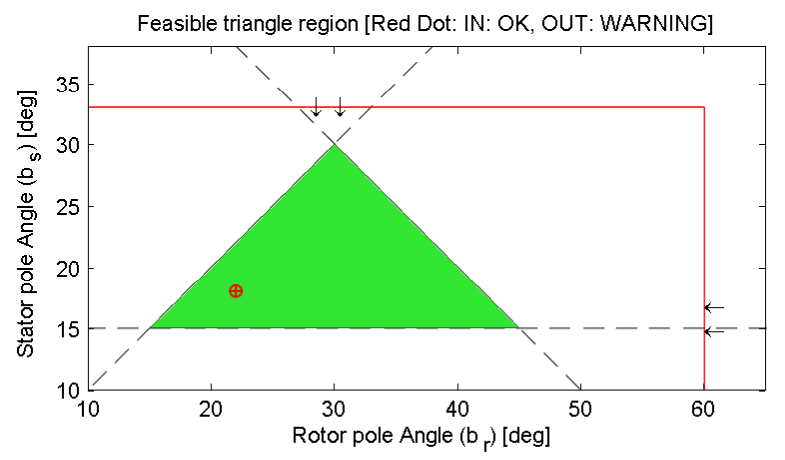

Figure 10. SRM Feasible Region Sizing Chart. Allows to determine if motor complies with 5 conditions between rotor and stator pole angles.

Also an efficiency map is generated as a preliminary representation of the motor behavior. Efficiency map is a representation of torque vs mechanical speed chart with efficiency of each point following a color gradient depending on its value (Figure 11). This map is able to take into account copper and iron losses. The map requires the following information from pre-design: inductances; phase voltage; field; average flux density and mass of each part where iron losses are considered (this is obtained thanks to the Moments of inertia and masses function); phase resistance and a maximum speed and current. The efficiency map computes, in a series of concatenated loops, the equivalent circuit of the machine taking into account both phase resistance in series and iron losses in a parallel branch. Iron losses are taken into account by means of an implementation of Bertotti's equation [10]. To calculate losses 
following that method three elements are required: average field densities are found via the flux densities determined for each part, volumes (masses) are computed in a previous stage: Masses and Moments of Inertia function, and finally coefficients are found once material for each part has been selected or can be introduced manually.

\section{Future work}

The aim of any pre-design tool is to reduce the required information designer must provide in order to achieve a feasible design. This is directly related to the number of inputs required in the first iterations of the design, before entering into a refining process. On the other hand by means of further layers in the design predesign tools can provide even richer sets of data without landing in the long simulation times of FEA solutions.

It should be able to handle several winding layers and fractional slotting and present the results graphically. One very complete free solution in this regard is EMETOR online SMPMSM tool [11].

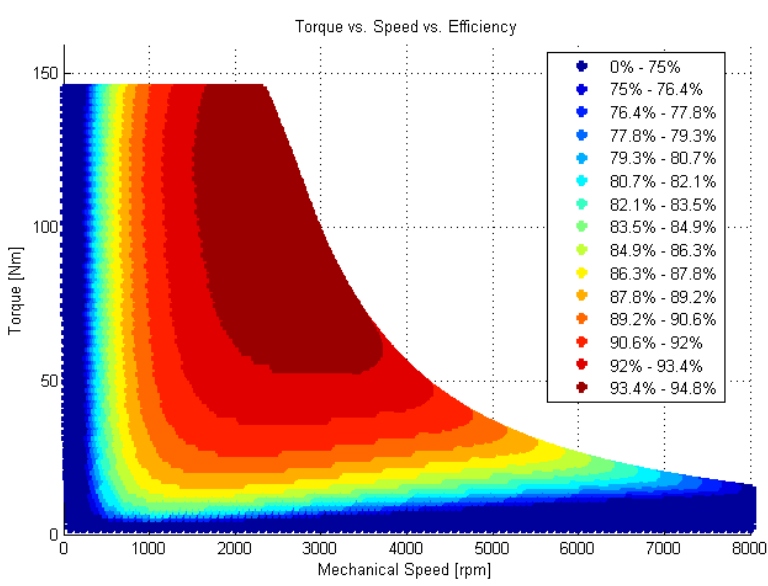

Figure 11. Torque vs. Speed vs. Efficiency representation (a.k.a. Efficiency map) for an IPM machine delivered by the pre-sizing tool. Data is calculated under Modelica, plotted by GUI.

Also the introduction of thermal restrictions by means of a thermal model would also optimize the design process and prepare the models for even more complex simulation scenarios. Thermal model should be able to interact with resistance values during predesign process and also able to specify losses and heat transfer with the surroundings.

For the second proposed approach a reluctance network of each machine based on an electro-magnetic model of the machine would provide the golden mean between a basic pre-design and a FE analysis. Reluctance network should provide variables such as torque ripple frequencies and magnitudes, Back Electro Motive Force shape, airgap flux shape, both taking into account armature reaction. And several other variables usually reserved to FE simulations. It also should be able to report variations in performance and behavior based on different control approaches given by Modelica implemented systems of such nature.

\section{Conclusions}

This tool provides an easy and intuitive way to generate machine designs in order to provide, to further design steps, seed values to work with. Also, by means of a refinement process, obtain close to final machine properties to use into system model design. It as well helps to determine pre-design inconsistencies and evaluate machine properties by means of the debug data in console, the graphical aids during design process and graphical representation of outputs.

Thanks to the inclusion of the efficiency map a preliminary behavior of the machine can also be evaluated before setting it up in any system.

\section{Acknowledgements}

Modelica implementation of this pre-design tool is part of Modelica Library of Detailed Magnetic Effects in Rotating Machinery (MAGMOLIB, SP1-JT1-CS2013-01, GA-620087) project managed by the German Aerospace Center (DLR) and developed by MCIA research center a part of the CLEANSKY partnership, a Public Private Partnership between the European Commission and the aeronautical industry.

\section{References}

[1] M. R. Kuhn, A. Griffo, W. Jiabin, and J. Bals, "A components library for simulation and analysis of aircraft electrical power systems using Modelica," in Power Electronics and Applications, 2009. EPE '09. 13th European Conference on, 2009, pp. 1-10.

M. R. Kuhn, "Advanced generator design using pareto-optimization," in Power Electronics and Drive Systems (PEDS), 2011 IEEE Ninth International Conference on, 2011, pp. 1061-1067.

[3] J. Yang, "A novel modelica based design platform for switched reluctance drive systems," in Electrical Machines and Systems (ICEMS), 2014 17th International Conference on, 2014, pp. 3302-3308.

[4] C. Kral, A. Haumer, and R. Wöhrnschimmel, "Extension of the FundamentalWave Library towards Multi Phase Electric Machine Models," ed, 2014.

[5] The Clean Sky Joint Technology Initiative. Available: http://www.cleansky.eu/

[6] (2015). Actuaction2015. Available: http://www.actuation2015.eu/

[7] C. Schlegel and R. Finsterwalder, "Automatic Generation of Graphical User Interfaces for Simulation of Modelica Models," presented at the Modelica Conference 2001, 2011.

[8] J. R. a. M. Hendershot, T.J.E., Design of brushless permanent-magnet motors. Hillsboro, $\mathrm{OH}$ : Oxford : Magna Physics Pub: Motor Design Books LLC, 1994.

[9] R. Jordi-Roger, G. Antoni, and R. Luís, "A computer experiment to simulate the dynamic behaviour of 
Multi Electrical Machine Pre-Design Tool with Error Handling and Machine Specific Advanced Graphical Design Aid Features Based on Modelica

electric vehicles driven by switched reluctance motors," vol. 51, ed: International Journal of

Electrical Engineering Education, 2014, pp. 368-382.

[10] M. Chunting, G. R. Slemon, and R. Bonert, "Modeling of iron losses of permanent-magnet synchronous motors," Industry Applications, IEEE Transactions on, vol. 39, pp. 734-742, 2003.

[11] R. I. o. Technology. (2008). EMETOR. Available: www.eme.ee.kth.se/emetor 